\title{
Theoretical Consideration of Heat Flux Distribution of Arc Driven by AC Magnetic Field
}

\author{
Takeo YAMAMOTO, ${ }^{1)}$ Naomi MATSUMOTO, ${ }^{1)}$ Masaya SUGIMOTO, ${ }^{1)}$ Seiichi SUDO,, ${ }^{1)}$ Takehiko TOH ${ }^{2 l}$ \\ and Koichi TAKEDA ${ }^{11}$
}

1) Department of Machine Intelligence and System Engineering, Akita Prefectural University, 84 Tuchiya Ebinokuchi, YuriHonjo, Akita 015-0055 Japan. 293-8511 Japan.

\author{
2) Technical Development Bureau, Nippon Steel Corporation, 20-1 Shintomi, Futtu, Chiba
}

(Received on July 1, 2009; accepted on September 7, 2009)

\begin{abstract}
A magnetically driven arc is produced by imposing an external magnetic field to a transferred arc. Its width is determined by the amplitude of its oscillatory motion. Its heat flux distribution can be controlled by the waveform of the magnetic field. Prediction of the heat flux distribution on the anode is necessary to apply the magnetically driven arc in industrial fields.

This paper describes the theoretical examination of the magnetically driven arc. A one-dimensional heat source with a Gaussian distribution is assumed to predict the heat flux distribution within the amplitude. First, the heat flux variation according to the magnetic flux density was examined under a DC magnetic field. Based on that investigation, the heat flux distributions in various AC magnetic fields were considered within the oscillatory amplitude.
\end{abstract}

KEY WORDS: transferred arc; heat flux; gaussian distribution; magnetic field.

\section{Introduction}

Plasma arcs have been widely used for cutting or welding metals because they can supply a dense energy flow in a small area of an arc spot. However, it is not a convenient heat source to treat a wide area. To expand a plasma arc, various trials have been attempted. ${ }^{1-4)}$ Takeda et $a .^{3,4)}$ developed a magnetically driven arc by imposing an alternating magnetic field perpendicular to a transferred arc. The arc oscillates because of the interaction between the electric arc current and the external magnetic field. Typical plasma arc profiles are presented in Fig. 1. An arc in no magnetic field, as shown in Fig. 1(a), deformed by a DC magnetic field, as portrayed in Fig. 1(b). By imposing an AC magnetic field, the arc oscillates as depicted in Fig. 1(c).

For practical applications of such a magnetically driven arc, precise understanding of the heat flux distribution is necessary. Experimental observation by Yamamoto et al. ${ }^{5)}$ revealed that the heat flux decreased near the edge of the oscillatory amplitude. Toh et al. ${ }^{6}{ }^{6}$ has attempted to predict the heat flux distribution theoretically using a 3D MHD model. The present study develops a simple but practical model to predict heat flux variations with various waveforms of the imposed magnetic field, using the assumption of a one-dimensional heat source.

\section{Theoretical Consideration of the Heat Flux Varia- tion with DC Magnetic Flux Density}

\subsection{Displacement of the Arc Root by Imposing Mag- netic Flux Density}

A schematic of the setup used to produce a magnetically driven arc is presented in Fig. 2. As depicted therein, the following coordinate system $(x, y, z)$ is used in this study. A plasma torch is located on the $z$ axis; the anode expands in the $x-y$ plane. An external magnetic field is generated perpendicular to the arc by DC electric current running through a two-turn rectangular field coil. The magnetic field is imposed in the direction of $x$. The arc current's interaction with the external magnetic field results in arc movement in the $y$ direction. Without any imposed magnetic field, the center of the arc root is located at the origin
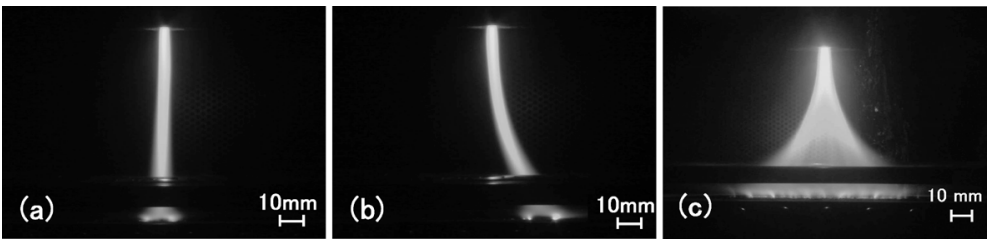

Fig. 1. Various plasma arcs: in no magnetic field (a), in a DC magnetic field (b), and in an AC magnetic field (c). 


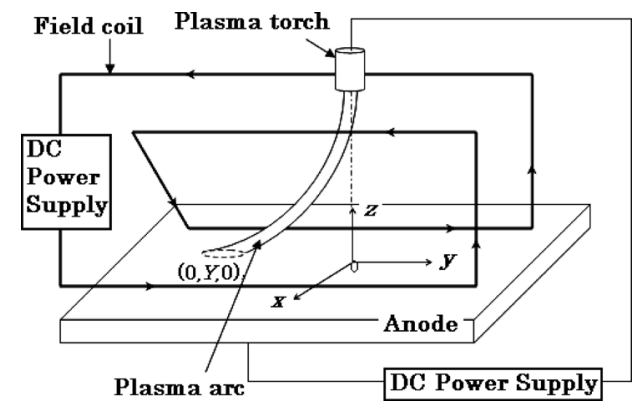

Fig. 2. Schematic illustration of the system to produce a magnetically driven arc.

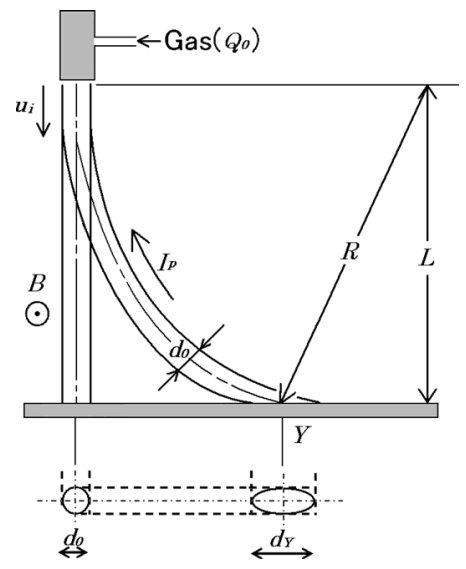

Fig. 3. Schematic illustration of the arc root deformation.

$(0,0,0)$. With a magnetic field, it moves to $(0, Y, 0)$.

In a uniform magnetic field, it is known that displacement $Y$ is expressed as ${ }^{3)}$

$$
Y=R-\sqrt{R^{2}-L^{2}}
$$

where $L$ denotes the standoff distance between a torch orifice and an anode plane. A radius of curvature $R$ varies inverse proportionally to an imposed magnetic flux density $B$.

\subsection{Deformation of the Arc Root with Its Displace- ment}

The arc at $Y=0$ is assumed to be cylindrically symmetric. As presented in Fig. 3, the cross section of the arc on the anode changes from a circle with diameter $d_{0}$ to an ellipse with minor diameter $d_{0}$ and major diameter $d_{Y}$ in an imposed magnetic field.

Considering Eq. (1), geometrical considerations engender the following relation:

$$
\frac{d_{Y}}{d_{0}}=\frac{L^{2}+Y^{2}}{L^{2}-Y^{2}}
$$

The heat flux distribution function in the arc root is introduced as $h_{Y}(x, y)$, where subscript $Y$ represents that its center position is located at $Y$. As described later, the heat flow from the arc to the anode remains constant independent of the imposed magnetic flux density. Under such a condition, the following ratio on the heat flux is satisfied:

$$
\frac{h_{Y}(0, Y)}{h_{0}(0,0)}=\frac{d_{0}}{d_{Y}}=\frac{L^{2}-Y^{2}}{L^{2}+Y^{2}}
$$

In that equation, $h_{0}(0,0)$ and $h_{Y}(0, Y)$ respectively denote the heat flux at the center of the arc root in no magnetic

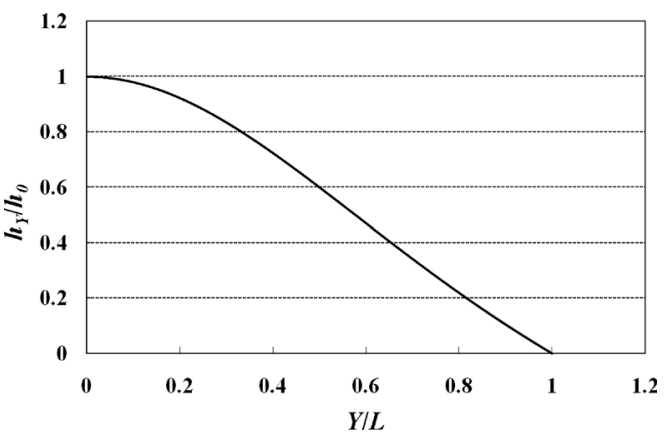

Fig. 4. Variation of heat flux at the center of the arc root with $Y$.

field and that in imposed magnetic field, according to the definition of $h_{Y}(x, y)$.

As portrayed in Fig. 4, the heat flux decreases concomitantly with increased $Y$ or $B$.

\subsection{Transformation of 2D Heat Flux Distribution to 1D Heat Flux Distribution}

To simplify the theoretical considerations, the two-dimensional heat flow is transformed into one-dimensional heat flow, regarding the arc root as a linear heat source varying its length with the imposed magnetic flux density. One dimensional heat flux is expected to satisfy the following relation.

$$
H_{Y}(y)=\int_{-\infty}^{+\infty} h_{Y}(x, y) d x
$$

In no magnetic field, it is expressed as $H_{0}(y)$ because the arc root center is located at $Y=0$. Because the heat flux has its maximum value at the center of the arc root and because its value decreases concomitantly with increased distance from the center, ${ }^{7}$ the authors assume the following Gaussian distribution function for a one-dimensional heat source in no magnetic field:

$$
H_{0}(y)=\frac{P_{0}}{\sigma_{0} \sqrt{2 \pi}} \exp \left(-\frac{y^{2}}{\sigma_{0}^{2}}\right)
$$

where $P_{0}$ and $\sigma_{0}$ respectively signify the total heat flow from the arc to the anode and a measure of the width in the Gaussian distribution function. In the imposed magnetic field, the distribution function for one-dimensional heat source whose center is located at $Y$ is expressed as

$$
H_{Y}(y)=\frac{P_{0}}{\sigma_{Y} \sqrt{2 \pi}} \exp \left(-\frac{(y-Y)^{2}}{\sigma_{Y}^{2}}\right)
$$

The width in Eq. (6) varies with $Y$. Regarding the relation between $\sigma_{Y}$ and $\sigma_{0}$, it is reasonably assumed that

$$
\frac{\sigma_{Y}}{\sigma_{0}}=\frac{d_{Y}}{d_{0}}=\frac{L^{2}+Y^{2}}{L^{2}-Y^{2}}
$$

Therefore, Eq. (7) can be rewritten as follows using the relation presented in Eq. (6).

$$
H_{Y}(y)=\frac{d_{0}}{d_{Y}} \cdot H_{0}\left(\frac{d_{0}}{d_{Y}}(y-Y)\right)
$$




\section{Determination of and Confirmation of the Assump- tions' Validity}

\subsection{Experiment Using a Calorimeter}

To determine $\sigma_{0}$ and to confirm the validity of the assumptions used in the theoretical model, an experiment was conducted using a similar experimental arrangement to that shown in Fig. 2. A transferred arc was produced between a plasma torch with a tungsten cathode and a water-cooled copper anode. The standoff distance between the torch orifice and the anode was fixed at $70 \mathrm{~mm}$. A power supply was connected to the torch; the anode was operated in a constant current mode. The arc current was adjusted at $130 \mathrm{~A}$. The open circuit voltage of the power supply was $280 \mathrm{~V}$. Argon gas was fed to the torch as a plasma forming gas at the flow rate of $4.5 \times 10^{-4} \mathrm{~kg} / \mathrm{s}$. An external magnetic field was generated perpendicular to the arc using DC electric current through a two-turn rectangular field coil. The maximum magnetic flux density in the coil center was $2.22 \mathrm{mT}$ at the coil current of $300 \mathrm{~A}$.

In this experiment, a special anode assembly was developed to estimate the heat flux distribution in the arc root. As depicted in Fig. 5, it consisted of three water-cooled copper blocks. The 10-mm-wide, 10-mm-thick and 100$\mathrm{mm}$-long center block is designated as a calorimeter hereinafter. The calorimeter was thermally insulated from the others. It was moved by a motor drive mechanism in the direction of the arc displacement at $0.33 \mathrm{~mm} / \mathrm{s}$, at that driving speed, it was confirmed that the difference between the observed heat flow and that of at steady state was negligible small. The heat flow to the calorimeter was estimated as the temperature increase $\left(T_{\mathrm{out}}^{\mathrm{c}}-T_{\mathrm{in}}^{\mathrm{c}}\right)$ in the cooling water flowing through this block.

We must bear in mind that the distribution of the heat

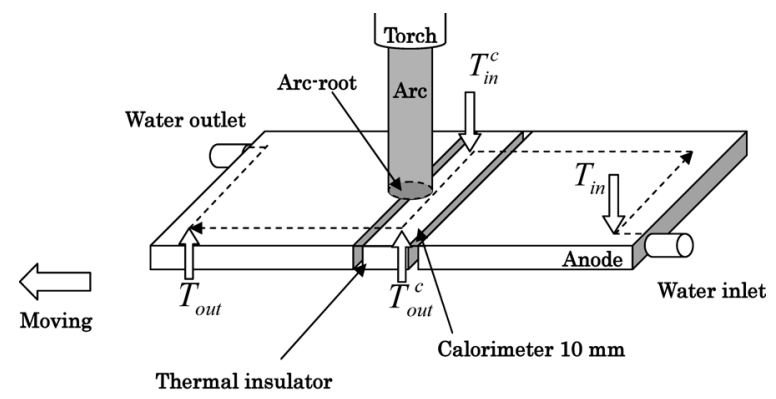

(a) Anode assembly and a calorimeter

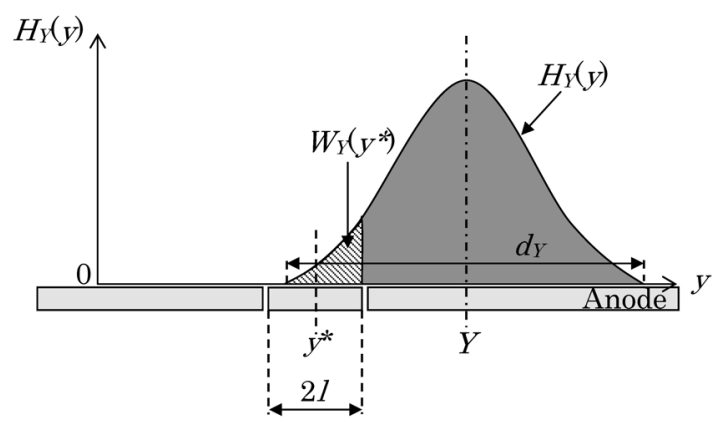

(b) Heat flow to the calorimeter

Fig. 5. Anode assembly (a) and schematic illustration of the heat flow to the calorimeter (b). flux in the anode root can not be measured directly by the calorimeter. As portrayed in Fig. 5(b) the heat flow into the calorimeter having the width of $2 l, W_{Y}\left(y^{*}\right)$ is expressed in the following equation:

$$
W_{Y}\left(y^{*}\right)=\int_{y^{*-l}}^{y^{*+l}} H_{Y}(y) d y
$$

where $y^{*}$ represents the center position of the calorimeter, and $H_{Y}(y)$ denotes the one-dimensional heat flux distribution function in the arc root centered at $Y$.

\subsection{Determination of $\sigma_{0}$}

Without an imposed magnetic field, the heat flow into the calorimeter located at $y^{*}=0$ is expressed as

$$
W_{0}(0)=\int_{-l}^{+l} H_{0}(y) d y=\frac{P_{0}}{\sigma_{0} \sqrt{2 \pi}} \int_{-l}^{+l} \exp \left(-\frac{y^{2}}{\sigma_{0}^{2}}\right) d y
$$

The total heat flow $P_{0}$ in Eq. (10) was determined according to the measurement of temperature difference $\left(T_{\text {out }}-T_{\text {in }}\right)$ through the anode assembly. Experimental measurements revealed that $P_{0}$ kept constant independent of the magnetic flux density. In the present experimental condition, the obtained $P_{0}$ was $7.2 \mathrm{~kW}$. Without any imposed magnetic field, the heat flow to the calorimeter at $y^{*}=0$ was $3.3 \mathrm{~kW}$. From Eq. (10), $\sigma_{0}$ was estimated as $\sigma_{0}=8.2 \mathrm{~mm}$.

\subsection{Confirmation on the Validity of the Assumptions}

Theoretical variation of $W_{Y}\left(y^{*}\right)$ can be calculated using the experimental data of $\sigma_{0}$ and $P_{0}$ obtained in Sec. 3.2, in two different cases of $Y$, theoretical predictions of $W_{Y}\left(y^{*}\right)$ were compared with measured variations of the heat flow to the calorimeter.

Figure 6 presents a comparison between theoretical predictions and experimental results for various $y^{*}$ for $Y=0$. The theoretical predictions closely match the experimental profile. After the collision on the anode, hot plasma gas expands along the anode surface, heat flow from the expanded plasma gas may cause the difference between experiment and theory.

A similar comparison at $Y=-29 \mathrm{~mm}$ is portrayed in Fig. 7. In this case, the agreement was also good, meaning that the assumptions used in the theoretical model are confirmed as valid.

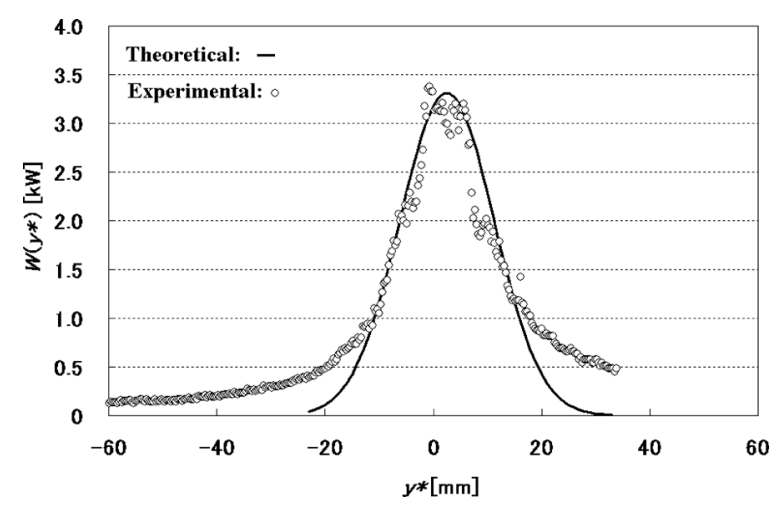

Fig. 6. Comparison between the theoretical result and experimental result without external magnetic field. 


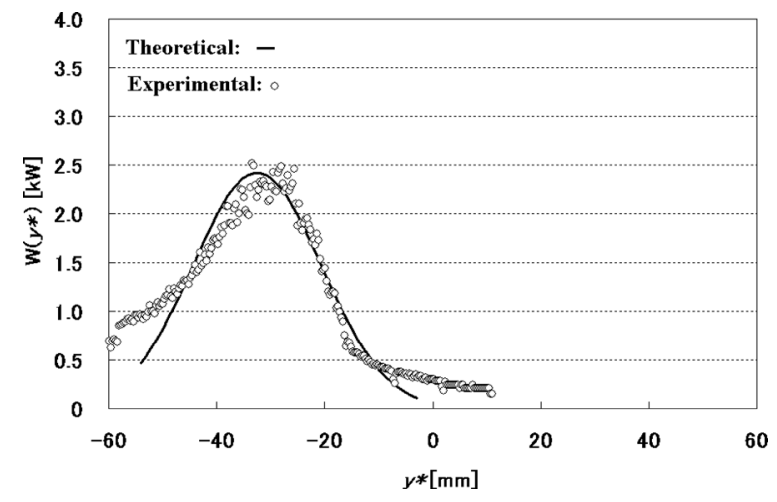

Fig. 7. Comparison between the theoretical result and experimental result with a DC magnetic field at $Y=-29$.

These comparisons confirmed that the one-dimensional mode with a Gaussian distribution function is useful to predict variation of the heat flux with the imposed magnetic flux density, in spite of the rough assumptions used.

\section{Theoretical Prediction on the Heat Flux Distribu- tion in AC Magnetic Field}

If the imposed $\mathrm{AC}$ magnetic flux density $(B)$ is expressed in the following arbitrary form as

$$
B(t)=B_{0} f(t)
$$

then the arc root center position $(Y)$ is reasonably assumed to be in a similar form of

$$
Y(t)=Y_{0} f(t)
$$

where $B_{0}$ and $Y_{0}$ respectively represent the amplitude of the AC magnetic flux density and that of the arc root movement. Function $f(t)$ represents the waveform varying between -1 and 1 . In a small distance $d y$ for a short time interval $d t$, the heat flow around a position $y$ and a time $t$ is defined as shown below.

$$
d q(y, t)=H_{Y}(y) d y \cdot d t .
$$

During the half period of the AC magnetic field, the heat flow into $d y$ can be expressed as

$$
d Q(y)=\left\{\int_{0}^{\tau} d q(y, t) d t\right\} d y=\left\{\int_{-Y_{0}}^{Y_{0}} H_{Y}(y) \frac{d t}{d Y} d Y\right\} d y
$$

where $\tau$ represents the half period of the imposed field. Then the heat flux averaged over the half period of the AC field is obtained as shown in Eq. (15).

$$
\langle H(y)\rangle=\frac{1}{\tau} \frac{d Q(y)}{d y}=\frac{1}{\tau} \int_{-Y_{0}}^{Y_{0}} H_{Y}(y) \frac{d t}{d Y} d Y
$$

In that equation, $\langle H(y)\rangle$ can be calculated numerically. For example, if the imposed AC magnetic field varies in a sinusoidal form, Eq. (12) can be expressed as shown below.

$$
Y=Y_{0} \cos \left(\frac{\pi}{\tau} t\right)
$$

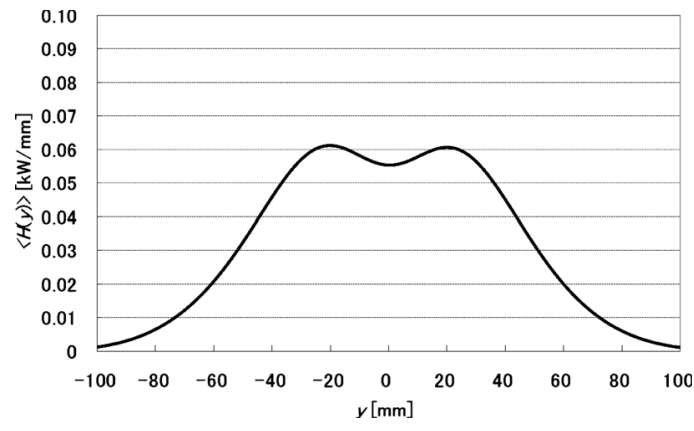

Fig. 8. Heat flux distribution with a sinusoidal magnetic field.

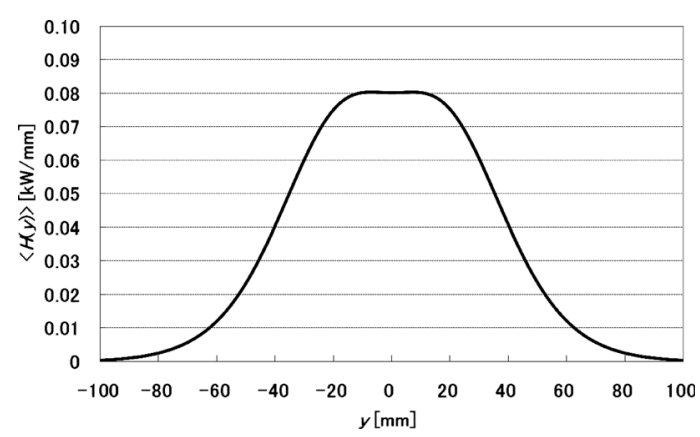

Fig. 9. Heat flux distribution with a triangular magnetic field.

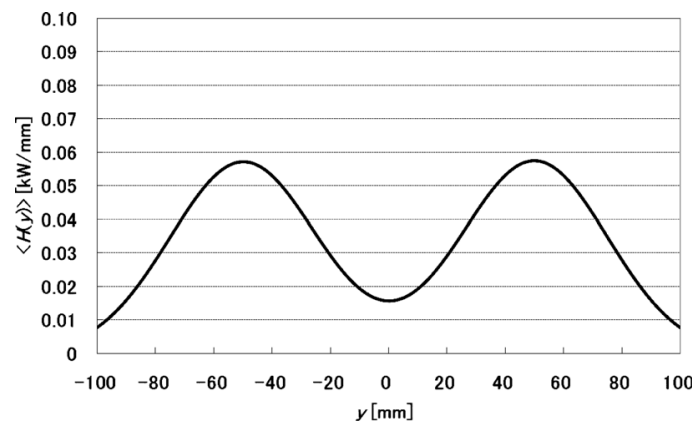

Fig. 10. Heat flux distribution with a rectangular magnetic field.

Consequently, Eq. (16) can then be rewritten as

$$
\langle H(y)\rangle=\pi \int_{-Y_{0}}^{Y_{0}} H_{Y}(y) \frac{d Y}{\sqrt{Y_{0}^{2}-Y^{2}}}
$$

Numerical calculations were conducted for three different AC waveforms. In all cases, $Y_{0}, P_{0}$, and $\sigma_{0}$ were assumed, respectively, as $4.0 \times 10^{-2} \mathrm{~m}, 7.2 \mathrm{~kW}$ and $8.2 \times 10^{-3} \mathrm{~m}$. Figure 8 shows a heat flux distribution in the $\mathrm{AC}$ magnetic field with a sinusoidal waveform. Figures 9 and $\mathbf{1 0}$ respectively show the heat flux distribution in a triangular magnetic field and that in a rectangular magnetic field.

Comparison of Fig. 8, Fig. 9 and Fig. 10 clearly shows that the heat flux distribution depends on the external magnetic waveform, meaning that the heat flux distribution on the anode can be controlled by changing the waveform of the imposed magnetic field.

\section{Conclusions}

Theoretical examinations were performed to predict the heat flux distribution of a magnetically driven arc. Conclu- 
sions can be summarized as follows.

(1) Experimental results revealed that transformation of the $2 \mathrm{D}$ heat flux inside the arc root to 1D heat flux was useful to simplify the model and estimate a heat flux distribution on the anode.

(2) Results of the experiment confirmed that the assumption of Gaussian distribution was reasonable for the 1D arc root model.

(3) Numerical calculations revealed that the heat flow profile along the oscillatory motion was controlled by changing the waveform of the imposed magnetic field.

\section{Acknowledgement}

This work was financially supported by the Ministry of Economy, Trade and Industry of Japan.

\section{Nomenclature}

$B$ : Magnetic flux density (T)

$B_{0}$ : Amplitude of magnetic flux density (T)

$H_{Y}(y)$ : One-dimensional heat flux in DC magnetic field $(\mathrm{W} / \mathrm{m})$

$\langle H(y)\rangle$ : Time averaged heat flux in AC magnetic field $(\mathrm{W} / \mathrm{m})$

$h_{0}$ : Heat flux at the center of the arc root in no magnetic field $\left(\mathrm{W} / \mathrm{m}^{2}\right)$

$h_{Y}$ : Heat flux at the center of the arc root in imposed magnetic field $\left(\mathrm{W} / \mathrm{m}^{2}\right)$

$L$ : Standoff distance between arc torch and anode (m)

$P_{0}$ : Total heat flow from the arc to the anode (W)

$d Q(y)$ : Heat transferred to the small distance $d y$ during a half period $(\mathrm{J})$

$R$ : Radius of curvature of the deflected arc (m)

$T_{\text {in }}$ : Temperature of cooling water at the inlet of the anode $(\mathrm{K})$

$T_{\text {out }}$ : Temperature of cooling water at the outlet of the anode $(\mathrm{K})$
$T_{\text {in }}^{\mathrm{c}}$ : Temperature of cooling water at the inlet of the calorimeter $(\mathrm{K})$

$T_{\text {out }}^{\mathrm{c}}$ : Temperature of cooling water at the outlet of the calorimeter $(\mathrm{K})$

$Y$ : Position of arc root center (m)

$Y_{0}: \quad$ Amplitude of the arc center oscillation (m)

$W_{Y}\left(y^{*}\right): \quad$ Heat flow to the calorimeter at $y=y^{*}(\mathrm{~W})$

$d_{0}$ : Minor diameter of the arc root $(\mathrm{m})$

$d_{Y}:$ Major diameter of the arc root $(\mathrm{m})$

$f(t)$ : Waveform varying with time $(-)$

$l$ : Half width of the calorimeter $(\mathrm{m})$

$d q(y, t)$ : Heat transferred to a small distance $d y$ for short time interval $d t(\mathrm{~J})$

$t:$ Time (s)

$x, y, z:$ Coordinate $(\mathrm{m})$

$y^{*}:$ Center position of the calorimeter $(\mathrm{m})$

$\sigma_{0}$ : Measure of the width in Gaussian function for $Y=0(\mathrm{~m})$

$\sigma_{Y}$ : Measure of the width in Gaussian function for $Y=Y(\mathrm{~m})$

$\tau$ : Half period of the oscillation (s)

\section{REFERENCES}

1) Y. Arata and H. Maruo: Technology Reports of the Osaka University, 22 (1972), 135.

2) J. E. Harry and D. Goodwin: Proc. of 4th Int. Conf. on Advances in Welding Processes, Weld. Inst., England, (1978), 181.

3) K. Takeda: Proc. Int. Workshop on Plasma Jet in the Development of New Materials Technology, VSP, Netherlands, (1990), 485.

4) K. Takeda: Generation of Magnetically Oscillating Plasma Arc and Its Properties, 16, J. High Temperature Soc., Osaka, (1990), 357.

5) T. Yamamoto, K. Takeda, T. Toh and J. Tanaka: Thin Solid Films, 515 (2007), 4228.

6) T. Toh, J .Tanaka, Y. Maruki, T. Yamamoto and K. Takeda: ISIJ Int., 45 (2005), No. 7, 947.

7) M. Tanaka, H. Terasaki, R. Narita, K. Kobayashi, H. Fujii and M. Ushio: Q. J. Jpn. Weld. Soc., 23 (2005), No. 3, 398. 